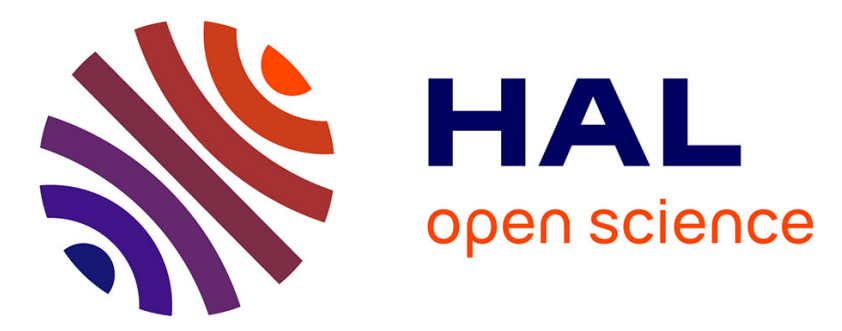

\title{
Interaction between perylene-derivated molecules observed by low temperature scanning tunneling microscopy
}

Loranne Vernisse, Olivier Guillermet, André Gourdon, Roland Coratger

\section{- To cite this version:}

Loranne Vernisse, Olivier Guillermet, André Gourdon, Roland Coratger. Interaction between perylene-derivated molecules observed by low temperature scanning tunneling microscopy. Surface Science: A Journal Devoted to the Physics and Chemistry of Interfaces, 2018, 669, pp.87-94. 10.1016/j.susc.2017.11.008 . hal-01804340

\author{
HAL Id: hal-01804340 \\ https://hal.science/hal-01804340
}

Submitted on 31 May 2018

HAL is a multi-disciplinary open access archive for the deposit and dissemination of scientific research documents, whether they are published or not. The documents may come from teaching and research institutions in France or abroad, or from public or private research centers.
L'archive ouverte pluridisciplinaire HAL, est destinée au dépôt et à la diffusion de documents scientifiques de niveau recherche, publiés ou non, émanant des établissements d'enseignement et de recherche français ou étrangers, des laboratoires publics ou privés. 


\title{
Interaction between perylene-derivated molecules observed by low temperature scanning tunneling microscopy
}

\author{
Loranne Vernisse ${ }^{1, *}$, Olivier Guillermet, André Gourdon, Roland Coratger \\ CEMES-CNRS, Université Paul Sabatier, 29 rue J. Marvig, BP 94347, F-31055 Toulouse, \\ France
}

\begin{abstract}
Derivative perylene molecules deposited on $\mathrm{Ag}(111)$ and on $\mathrm{NaCl}(001)$ ultrathin layers have been investigated using low temperature STM and NC-AFM. When the metallic substrate is held at ambient temperature during evaporation, the molecules form characteristic trimers on the $\mathrm{Ag}(111)$ surface and interact through their polar groups. Close to the steps, the molecules form linear structures and seems to stand side by side. On the other hand, after deposition on a substrate cooled at liquid helium temperature, single molecules are observed both on metal and on $\mathrm{NaCl}$. On the ultrathin insulator layers, the STM images present characteristic contrasts related to the molecular orbitals which favours the localization of aldehyde groups. In this case, the lateral molecular interactions may induce the formation of small assemblies in which the electronic levels are slightly shifted. A possible interpretation of this phenomenon is to take into account polar interactions and charge transfer between neighboring molecules.
\end{abstract} Keywords: STM; NC-AFM; Low Temperature; Perylene derivatives; Single Molecule; Molecular orbitals.

PACS: 68.37.Ef, 81.07.Nb

\footnotetext{
* Corresponding author

Email address: loranne.vernisse@univ-poitiers.fr (Loranne Vernisse)

${ }^{1}$ Current address: Institut P', UPR 3346 CNRS, Université de Poitiers, France
} 


\section{Introduction}

In order to create electronic devices based on molecules, one of the principal challenges is to be able to observe and control molecular assemblies. Thereafter, these assemblies will be used to study charge transfer for example between a sin-

5 gle molecule and a polymeric structure [1] or the choosen molecules will be the precursors of larger systems able to facilitate charge transfer [2, 3]. The molecular interactions within these assemblies is often driven by competitive forces either between the molecules in the assembly [4] or between the molecules and the substrate on which they are deposited [5] leading to different molecular lattices. Then, very different behaviours are expected between networks dominated by covalent bonding or by hydrogen interactions. In covalent assemblies, the intermolecular forces entirely determine the network structure since the chemical bonds are directive and very strong [6, 7]. Then, the molecular shape is used to produce stable networks of the desired geometry and reactivity depending of the used tecton [8]. On the other hand, hydrogen interactions are weaker and sometimes results in metastable structures in which the number of $\mathrm{H}$ bonds determines the final self assembly $[9$. However, these large molecular domains assembled by non covalent forces are technologically appealing since they can make possible the fabrication of nanostructures with controlled functionality, size and shape [10. Most of the studies reported so far concern large domains in which an important number of molecules are self-assembled and participate to the observed network [11. However, when the domains are very small, it appears interesting to study the interactions between the first molecules implied in the structure [12, 13, 14]. This may concern only a few molecules and in this case, their precise position in the native network can reflect the nature of the interactions involved in the first growth steps. Thus, the formation of these first structures can be used to predict the nature of the intermolecular interactions, an important point that cannot be always addressed in a complete monolayer.

Among the large number of possible candidates, perylene derivative molecules are objects of peculiar interest for various applications. Indeed, they are used in 
photonics and optoelectronics, where they can be used as pigments or colorant [15], in light-emitting diodes [16], transistors [17, photovoltaic cells [18, 19]. Optoelectronic characteristic of perylene derivative compounds are due to the $\pi$-conjugated system of its polyaromatic structure. On the other hand, it is 35 possible to change the characteristics of these molecules using appropriate peripheral groups, particularly if these groups are polar [20, 21].

In our investigations, we propose to use perylene derivative molecules as twodimensional models in the localization and interaction of local charges. Bearing this mind, we studied 3-perylene carbaldehyde molecules (PC) by STM and NC-

40 AFM on an $\mathrm{Ag}(111)$ surface and on ultrathin films of $\mathrm{NaCl}$. The $\mathrm{PC}$ molecule is a precursor of numerous perylene derivatives. Its chemical structure is presented Fig. 1. It is composed by a perylene group (in black) and an aldehyde group (in red). Although the molecule is neutral in gaz phase, its charge repartition is expected to be non uniform because of the peripheral group. Then, this molecule

45 is going to be used as a reference system in the exploration of molecular models with various local charges and their possible interactions.

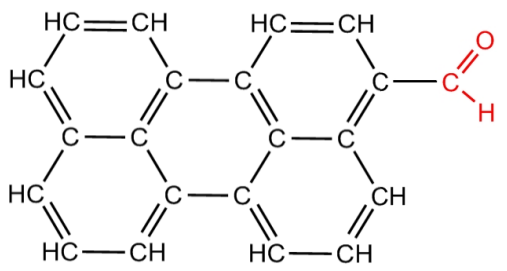

Figure 1: Chemical structures of 3-perylene carbaldehyde (PC) molecule

\section{Experimental details}

Scanning tunneling microscopy experiments have been performed at liquid helium temperature $(T=4.5 \mathrm{~K})$ on a commercial STM (LT Omicron) working with a base pressure of $2 \times 10^{-11}$ mbar. The $\mathrm{Ag}(111)$ crystal was cleaned by repeated $\mathrm{Ar}^{+}$bombardment cycles at $E=600 \mathrm{eV}$ followed by annealing at 
$800 \mathrm{~K}$ for one hour. Once the $\mathrm{Ag}(111)$ surface was clean, $\mathrm{NaCl}$ bilayers covering approximately $20 \%$ of the surface were grown onto the substrate held at $50^{\circ} \mathrm{C}$. The deposition time for such a coverage was about 2 minutes.

The synthesis of PC has been carried out according to [22]. The molecules were evaporated from a $\mathrm{W}$ filament $0.15 \mathrm{~mm}$ in diameter. This evaporator was first pumped by a turbo molecular pump during at least $12 \mathrm{~h}$. Then, before evaporation, the filament was submitted to high temperature outgassing cycles. Evaporations on the substrate held at room temperature were performed in the preparation chamber The filament was heated at 0.34 A during $30 \mathrm{sec}$.

Afterwards, the substrate was immediately transfered in the STM chamber to be cooled down at liquid helium temperature. The time between deposition and cooling process is estimated to be about 5 minutes, which is relatively large as compared to the typical diffusion time of molecules on metallic surfaces. One can therefore estimate that these conditions are sufficient to reach equilibrium in the formation of structures involving several molecules. Evaporations have also been conducted with the substrate held at liquid helium temperature, i.e. directly on the STM head through a small hole $1 \mathrm{~mm}$ in diameter drilled in one of the windows of the He cryostat. The filament was heated at $0.34 \mathrm{~A}$ during $2 \mathrm{~min}$. 70 The small solid angle supports very small evaporation rates with typical values in the 1/1000 monolayer range. These conditions were used for single molecule experiments in which thermal diffusion was inhibited. A particularity of PC molecules were their strong interaction with the different UHV chambers and also with the electronic connections in vacuum. This involved tip oscillations after several days of experiments and led to systematic baking at a temperature of $150{ }^{\circ} \mathrm{C}$ for $24 \mathrm{~h}$. Therefore, to prevent irremediable contamination, only low coverage experiments were performed. STM tips made of tungsten wires $0.2 \mathrm{~mm}$ in diameter were prepared by electrochemical etching and cleaned in UHV using direct current heating. Bias voltage was always applied to the sample.

The STM is equipped with a tuning fork of the qPlus sensor type allowing noncontact atomic force microscopy. The resonance frequency of the tuning forks used in this study was typically $25 \mathrm{kHz}$ and their quality factor was in the 
10000 to 20000 range. NC-AFM/STM tuning fork tips were made of platinumiridium wires $0.05 \mathrm{~mm}$ in diameter cut by focused ion beam once glued to the cantilever.

\section{Results and discussion}

Evaporation at ambient temperature. The first molecular assemblies of PC have been observed after deposition of molecules with a submonolayer coverage on the $\operatorname{Ag}(111)$ surface held at room temperature. The surface was then cooled down

90 around $4.5 \mathrm{~K}$ for STM imaging. The molecules appears as small rectangles (1.1 $\mathrm{nm}$ wide and $1.35 \mathrm{~nm}$ length) with an apparent height of about $0.15 \mathrm{~nm}$ at $-1 \mathrm{~V}$. This geometry is close to the shape of the molecular structure. After deposition and diffusion on the flat terraces, small assemblies are produced. They are mostly composed of three molecules in a characteristic triangular organization 95 (Fig. 2a and 2b), where each monomer is oriented with an angle of $60^{\circ}$ with respect to the others in a clockwise or anticlockwise orientation, giving chiral assemblies (Fig. 2c). The characteristic and reproducible geometry of these trimers suggests an interaction due to the polar groups resulting from a specific organization of the $\mathrm{H}$ bonds including the aldehyde moieties and the hydrogen atoms of the three molecules. Because of the molecular asymmetry due to the $\mathrm{COH}$ part, chiral structures are favored and each molecule arranges to favor the maximum of interaction with its neighbors. Few assemblies involving more than three molecules were observed. For example, a couple of five molecule assemblies were observed on terraces (black arrow Fig. 2a). In this case, the behavior is always the same: extra molecules only come in addition to the triangular feature. However, such structures were mostly found next step edges (red arrow Fig. 2a), and around defects such as sulfur atoms always present on the $\operatorname{Ag}(111)$ surface (black arrow Fig. 2b).

Molecules can be adsorbed in a head to tail configuration (Fig. 2b). This only occurs along step edges under the influence of the well-known Smoluchowski effect and the induced local dipolar moment 23$]$. To confirm the role of the $\mathrm{COH}$ 


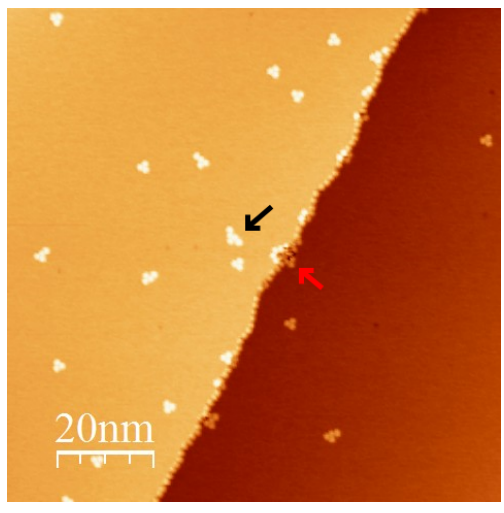

(a)

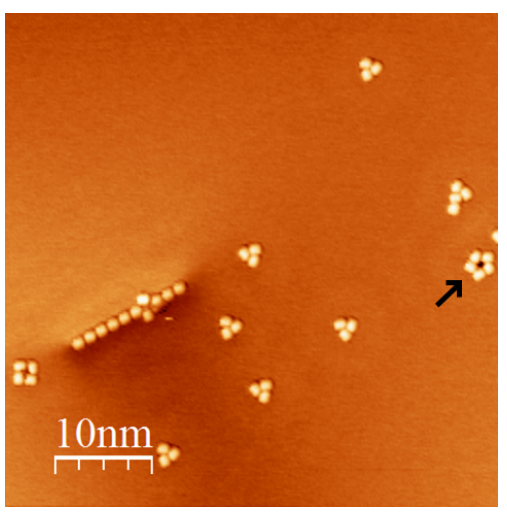

(b)
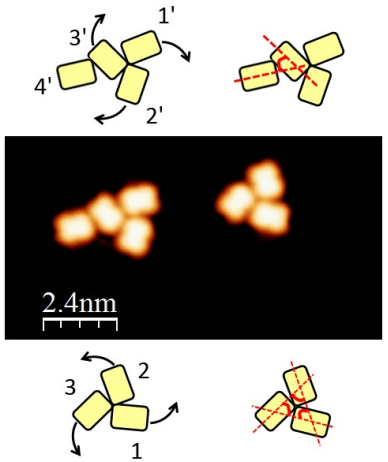

(c)

Figure 2: STM images of PC molecules (a) $100 \mathrm{~nm}$ x $100 \mathrm{~nm}$ (b) $50 \mathrm{~nm} \times 50 \mathrm{~nm}\left(\mathrm{~V}_{\text {bias }}=2 \mathrm{~V}\right.$, $\left.\mathrm{I}_{t}=2 \mathrm{pA}, \mathrm{T}=4.5 \mathrm{~K}\right)(\mathrm{c}) 12 \mathrm{~nm} \times 12 \mathrm{~nm} \mathrm{STM}$ image $\left(\mathrm{V}_{\text {bias }}=-1.5 \mathrm{~V}, \mathrm{I}_{t}=2 \mathrm{pA}, \mathrm{T}=4.5 \mathrm{~K}\right)$ of two molecular assemblies with their schematic representation respectively above and below the STM image.

moiety, the same type of perylene but with another peripheral group (perylene ethanoic acid: PEA) was also deposited on $\mathrm{Ag}(111)$ using the same experimental conditions (see supplementary materials). In this case, the structures were very different with a complete panel of assemblies including monomers, dimers, trimers,... In addition, no regular alignment along step edges were observed for PEA. This suggests that the PC trimer structures clearly result from the presence of the $\mathrm{COH}$ group while the regularity of the linear chain along the step implies a head to tail organization. 
The PC trimers were also investigated by NC-AFM. Before switching into the constant-height mode to obtain the $\Delta f$ images, the tip was positioned above the metal surface with tunneling parameters identical to those previously used for STM imaging (1.2 pA and $-400 \mathrm{mV})$. Thereafter, this height was kept constant and was not modified during $\Delta f$ imaging. The bias voltage of $-400 \mathrm{mV}$ was determined using $\Delta f(\mathrm{~V})$ curves and was chosen so that the contribution due to the electrostatic force was minimized. This value compensates the average contact potential difference (CPD) between tip and sample.

The NC-AFM image of the frequency shift at constant height is shown Fig. 3a. For clarity, the molecular structure has been superimposed in Fig. 3 b. The dark circle surrounding the trimer is mostly a consequence of attractive Van der Walls interactions and geometric effects of the structure. The carbon cycles are clearly distinguishable inside each monomer, especially inside monomer 1 . On this image, only perylene groups are resolved and the bond localization of the aldehyde group remains unclear. A first hypothesis consists to consider that the $\mathrm{HC}=\mathrm{O}$ bond is in a different horizontal plane, which could hide it. A second hypothesis, more realistic, is based on the close dependence between the tip height and the probed interactions [24, 25, 26]. Indeed, $\mathrm{C}=\mathrm{O}$ bonds were already observed by Zhang et al. in 8-hydroxyquinoline (8hq) molecules adsorbed on $\mathrm{Cu}(111)$ with a $\mathrm{CO}$ functionalized tip [27. They highlighted the fact that observation of the $\mathrm{C}=\mathrm{O}$ bond of this molecule requires to get closer to the surface to $30 \mathrm{pm}$. As a small change of the tip height during the image is therefore enough to change the type of interaction probed, it is possible that the $\mathrm{HC}=\mathrm{O}$ bond is not probed. Future experiments are going to be performed to study $\mathrm{C}=\mathrm{O}$ bonds as a function of the tip height. However, this image perfectly shows the chiral structure of the trimer and is also in good agreement with the structural model presented in Fig. 2C.

Evaporation at low temperature. The first experiments suggest that single molecules cannot be observed when the deposition is performed at ambient temperature. As diffusion induces the production of small clusters (stabilized by intermolec- 


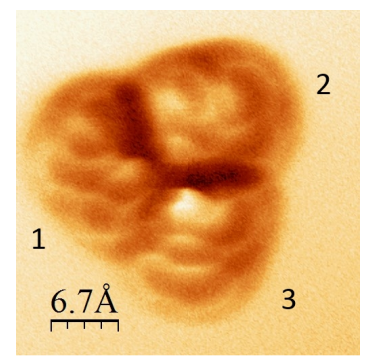

(a)
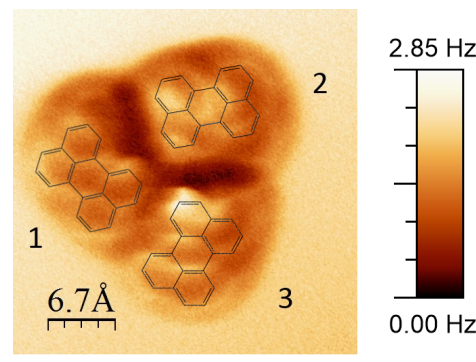

(b)

Figure 3: (a) Image of the frequency shift $\Delta f$ on a $\mathrm{PC}$ trimer ( $\mathrm{z}=\mathrm{cst}, \mathrm{V}_{\text {bias }}=-400 \mathrm{mV}$, $\mathrm{I}=1.2 \mathrm{pA}$ ) (b) Superposition of the chemical structure of perylene and the $\Delta f$ image while the sample is held at very low temperature.

For this and to preserve the electronic properties of the molecules, PC molecules were evaporated on a $\mathrm{Ag}(111)$ surface partly covered by $\mathrm{NaCl}$ islands. These islands generally have a thickness of two monolayers, denoted as coverage at a sample temperature held at $4.5 \mathrm{~K}$ led to individual molecules adsorbed both on the metallic surface and on the thin insulator.

The molecules present the same rectangular shape as previously described. However, the low temperature hinders diffusion and makes it possible to study single objects before the formation of trimers. Figure 4 shows PC molecules adsorbed on $\mathrm{Ag}(111)$ (A) and $\mathrm{NaCl}(\mathrm{B})$, and imaged at a positive bias voltage of $1.5 \mathrm{~V}$. If the molecules present the rectangular shape described above on $\mathrm{Ag}(111)$ (molecules 1), the same molecules observed at the same bias on $\mathrm{NaCl}$ exhibit a more complex geometry (molecules 2).

Once adsorbed on the ultra thin $\mathrm{NaCl}$ layer, the molecules present an interesting contrast that depends on the sign and value of the used bias voltage. Figs. $5 \mathrm{a}$ and $5 \mathrm{~b}$ show three $\mathrm{PC}$ imaged respectively at positive and negative bias voltages. Note that the molecule located on top has been slightly moved by the tip between the two images. When a negative voltage is applied, the molecule 


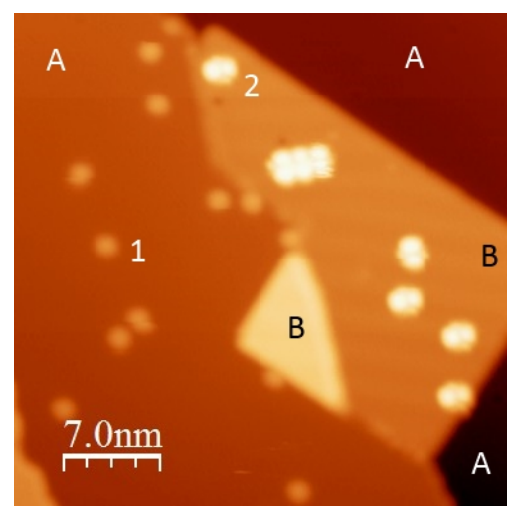

Figure 4: STM image after adsorption of PC molecules on a $\mathrm{Ag}(111)$ surface (A areas) partially covered by a bilayer of $\mathrm{NaCl}$ ( $\mathrm{B}$ area) $\left(\mathrm{V}_{\text {bias }}=1.5 \mathrm{~V}, \mathrm{I}=1 \mathrm{pA}, \mathrm{T}=4.5 \mathrm{~K}\right)$. The black square shows an assembly of 3 molecules.

shape looks similar to what is observed on $\operatorname{Ag}(111)$. Each molecule appears as a rounded rectangular structure. This image simply reveals the molecular skeleton. On the other hand, when a sufficient positive bias voltage is used, the apparent size of the molecule increases and the STM images reveal several lobes with different sizes and shapes. These lobes are always the same whatever the position of the molecules with respect to the $\mathrm{NaCl}$ surface. This is the case in Fig. 4 in which the single molecules appear identical although molecule labeled 2 is close to a small defect and another one close to a step edge. This means that a few angstroms away from these defects, the effects of these latter on the $\mathrm{NaCl}$ electronic structure such as the well-known $\mathrm{Cl}$ vacancy state reported by Repp et al. 28] do not affect the observed structures. However, we note in specific cases, a small shift of the threshold voltage for LUMO imaging when molecules are very close from a island edge. This shift is about $100 \mathrm{meV}$, does not appear systematically and depends on the molecule orientation with respect to the step direction.

These features results from the electronic structure of the molecule and the well-known decoupling properties of the ultra-thin $\mathrm{NaCl}$ layer.

$\mathrm{I}(\mathrm{V})$ spectra were also acquired on $\mathrm{PC}$ molecules adsorbed on $\mathrm{NaCl}$ and 


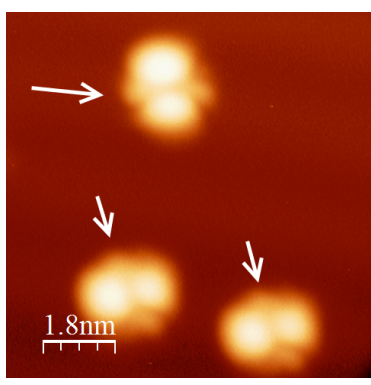

(a)

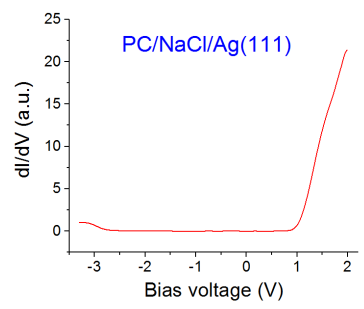

(c)

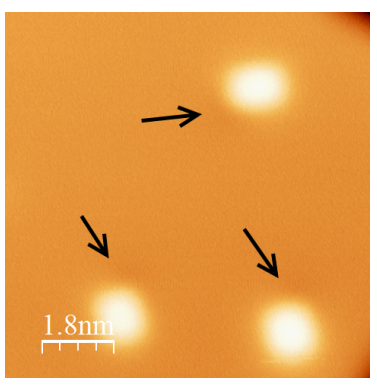

(b)

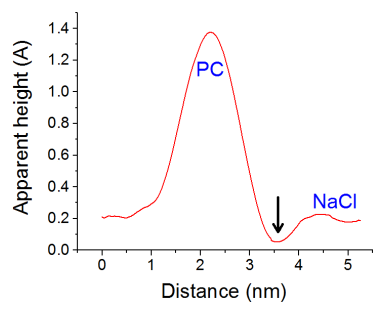

(d)

Figure 5: Observation of three $\mathrm{PC}$ molecules at: a) positive bias voltage $(1.5 \mathrm{~V})$. b) Negative bias voltage $(-1.5 \mathrm{~V})$. $(\mathrm{I}=1 \mathrm{pA}, \mathrm{T}=4.5 \mathrm{~K}))$. c) $\mathrm{dI} / \mathrm{dV}$ spectrum performed on a $\mathrm{PC}$ molecule adsorbed on $\mathrm{NaCl}$. d) Profile of a PC observed at negative bias voltage showing a small depression (also indicated by black arrows in (b).

presented after numerical derivative for clarity. A dI/dV spectrum acquired at the center of a PC molecule is presented Fig. 5c. No difference was noticed regarding the tip location above the molecule. It shows that at a positive voltage above typically $1.1 \mathrm{~V}$, the molecular LUMO state significantly contributes to the tunneling current (Fig. 5c). On the contrary, a small contribution from the HOMO state is observed for bias voltages below $-2.9 \mathrm{~V}$. At such voltages, clear STM cannot be perform on PC molecules. The difference between the two polarities is certainly due to the voltage drop that occurs between the molecule and the metal substrate and to the asymmetric position of the HOMO-LUMO gap around the Fermi energy. Note also that the orientation and position of the molecule with respect to the $\mathrm{NaCl}$ layer did not change the characteristic thresholds of the HOMO and LUMO states for single molecules except on the 
$\mathrm{NaCl}$ step egdes and for specific orientations of the molecules with respect to the step direction. This is consistent with the characteristics of the STM images discussed above and to the possible role of the local field generated by the steps that seems to shift the molecular orbital energies of about $100 \mathrm{meV}$. Thus, below the Fermi level, only the geometric shape of the molecule is observed and the images are similar to what is observed on the bare $\operatorname{Ag}(111)$ substrate. Nevertheless, a small feature appears as a small depression in one angle of each rectangular objects. This depression, about $15 \mathrm{pm}$ in depth, is highlighted in Figs. $5 \mathrm{~b}$ and $5 \mathrm{~d}$ by black arrows and is visible in all the STM images obtained at negative bias voltage.

We now focus our attention on the images produced above the LUMO onset. A small scale STM topography of this geometry and its computed counterpart are represented on Figs. 6a and 6b. The calculated image has been computed using the coupling between the atomic orbitals of each atom inside the molecule and a single s orbital of the tip (in this case a $\mathrm{Cu}$ atom is used for simplicity). Details of these calculations are given in supplementary materials.

The good agreement between the experimental images and the calculated ones suggests that this simple model is sufficient in this case to interpret the STM results. The observed and calculated structure consists of two main lobes, labeled 1 and 2, slightly asymmetric, and two antennas, the first composed by one lobe (3) and the second by two lobes (4 and 5). Figs. 6c and $6 \mathrm{~d}$ present the ball and stick representation of the molecule and the calculated contribution of the LUMO states. Both are oriented in the same direction as Figs. $6 \mathrm{a}$ and $6 \mathrm{~b}$. The comparison shows that it is perfectly possible to localize the aldehyde group as the lobe 3 on the STM image.

25 Using this imaging method above the LUMO threshold, the small depressions previously observed in Fig. $5 \mathrm{~b}$ can be located with respect to the molecular structure (lobe 3) and interpreted as the aldehyde moiety. Depressions on topographic STM images have previously been observed around molecules and can involve local charge transfer with oxygen atoms [29, 30]. To confirm that the observed dark contrast below the Fermi level could reflect a charge transfer, 


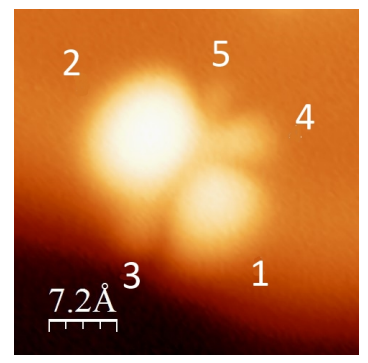

(a)

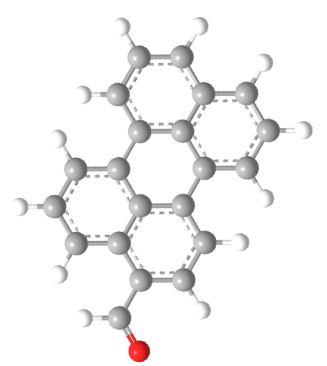

(c)

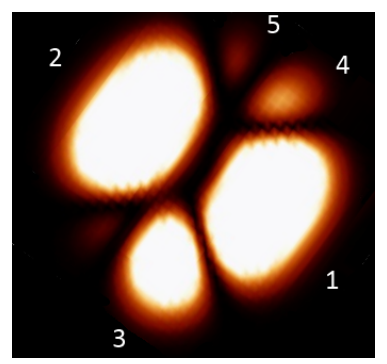

(b)

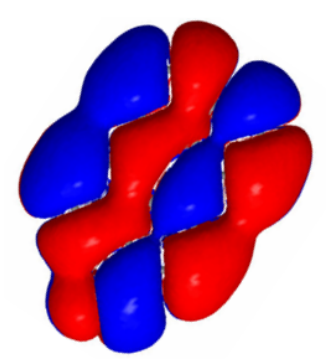

(d)

Figure 6: a STM images corresponding to the LUMO of a PC molecule adsorbed on a $\mathrm{NaCl}$ bilayer $\left(\mathrm{V}_{\text {bias }}=1.2 \mathrm{~V}, \mathrm{I}=1 \mathrm{pA}, \mathrm{T}=4.5 \mathrm{~K}\right) \mathrm{b}$ Computing image of the energy of the overlap of the HOMO and the 4 s orbital of a copper atom (tip) C Ball-and-stick representation of a PC molecule adsorbed on a $\mathrm{NaCl}$ bilayer. d Calculated positive and negative contributions of the LUMO. The molecule orientation is the same in the four images.

the local charge on the aldehyde group in the gas phase has been calculated using the MOPAC program. A value of $0.034|e|$ has been obtained, giving this molecule one of the largest dipole moment (3.339 D) among the perylene family. This leads to a charge transfer inside the molecule from the aldehyde group to the perylene part that tends to depopulate the $\mathrm{CHO}$ moiety. This latter then becomes slightly positively charged and produces a small decrease of the local density of occupied states. Therefore, at negative bias, the STM tip should probe this decrease as a small depression at the position of the $\mathrm{CHO}$ group, which is what is observed on the molecule. This result is also consistent for the three molecules presented in Figs. $5 \mathrm{a}$ and $5 \mathrm{~b}$ and has been observed on all the $\mathrm{PC}$ adsorbed on $\mathrm{NaCl}$. 
Interestingly, a few molecular assemblies can also be observed on $\mathrm{NaCl} / \mathrm{Ag}(111)$ in a head to tail configuration, as observed on the step edges on $\operatorname{Ag}(111)$. One of these assemblies, composed by three molecules, is visible in a black rectangle drawn in Fig. 4 and in a close-up view Fig. 7. Fig. 7a exhibits the characteristic shape and dimensions of PC molecules as observed at a low bias voltage. This simply suggests that they are not fragmented and look like those already presented in Fig. 2a. The three other images show that each of the three molecules presents the characteristic geometry of the LUMO state but for different bias voltages. Molecule 1 exhibits the typical LUMO geometry at 1.2 $\mathrm{V}$, while molecule 2 presents this shape at $1.3 \mathrm{~V}$ and molecule 3 at $1.4 \mathrm{~V}$.

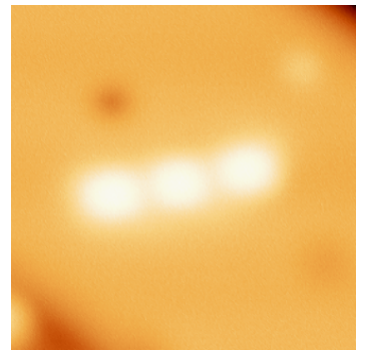

(a)

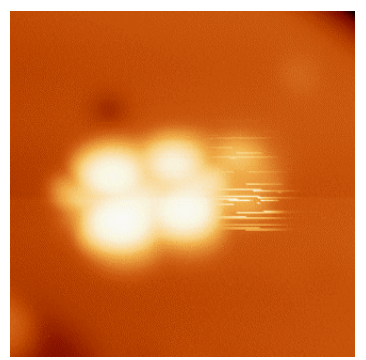

(c)

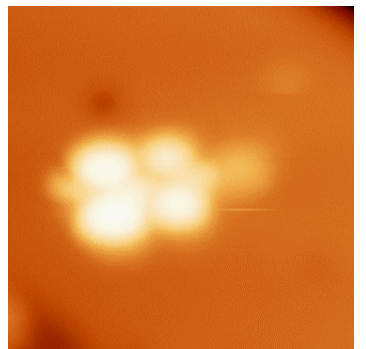

(b)

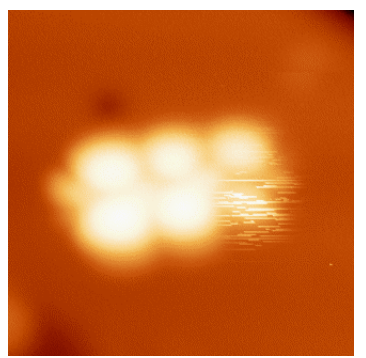

(d)

Figure 7: $\quad$ STM images $(6.8 \mathrm{~nm} \times 6.8 \mathrm{~nm})$ a self-assembly of $3 \mathrm{PC}$ molecules on $\mathrm{NaCl}(2 \mathrm{ML}) / \mathrm{Ag}(111)$ at different bias voltages. a) $\mathrm{V}_{\text {bias }}=0.9 \mathrm{~V}$ b $\mathrm{V}_{\text {bias }}=1.2 \mathrm{~V}$. c $\mathrm{V}_{\text {bias }}=1.3 \mathrm{~V}$. d) $\mathrm{V}_{\text {bias }}=1.4 \mathrm{~V}(\mathrm{I}=1 \mathrm{pA}, \mathrm{T}=4.5 \mathrm{~K})$.

Based on the calculations shown before and taking into account the previous discussion, it can be easily demonstrated that the PC molecules are assembled 
according to the schema presented Fig. 8. The left antenna of the assembly presents two lobes and is therefore on the opposite side of the aldehyde moiety of molecule 1. It has been previously observed that on a single PC molecule on $\mathrm{NaCl}$, the LUMO shaped geometry is totally reached at $1.3 \mathrm{~V}$. In the trimer, the LUMO state of central molecule 2 is observed at a constant energy of $1.3 \mathrm{eV}$ above the Fermi level $\mathrm{E}_{F}$. The central molecule 2 therefore exhibits the characteristic behavior of a single molecule. If $1.3 \mathrm{~V}$ is the difference between the substrate Fermi energy and the molecular LUMO state, this also shows that for the left molecule (1), this energy difference is lower of about $0.1 \mathrm{eV}$ as its LUMO shape is reached for a lower voltage $(1.2 \mathrm{~V})$. For the third molecule (3), the energy shift is opposite since the LUMO form is reached at a higher voltage $(1.4 \mathrm{~V})$. Thus, the two external molecules of the trimer differently behaves from the central one.

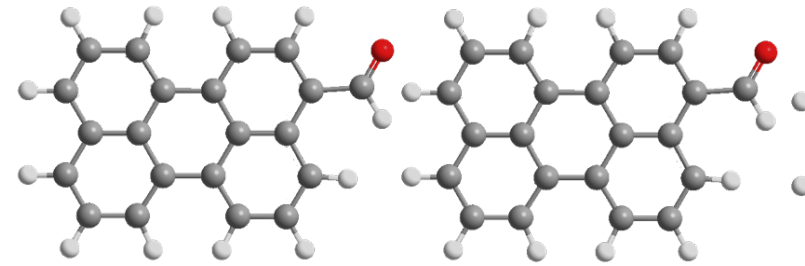

1
2

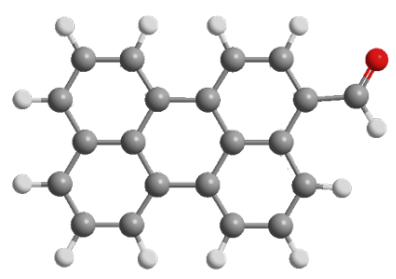

3

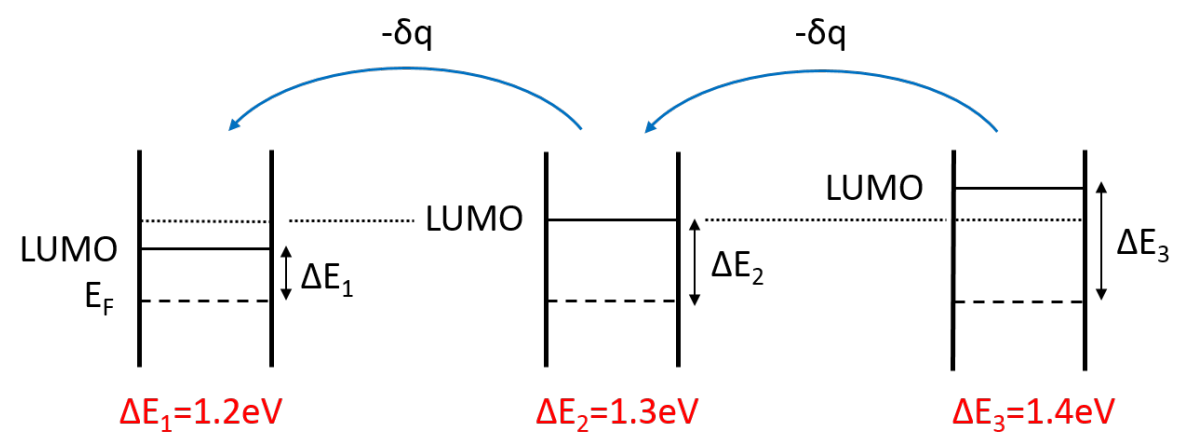

Figure 8: Schema representing the assembly of three PC molecules on $\mathrm{NaCl}(2 \mathrm{ML}) / \mathrm{Ag}(111)$

A first interpretation could be to invoke the adsorption location on the 
$\mathrm{NaCl}(001)$ surface [31]. Using Fig. 7a, an intermolecular distance of $1.34 \mathrm{~nm}$ has been measured. On the one hand, this distance is not consistent with the different adsorption sites on the $\mathrm{NaCl}$ surface (squared lattice with a parameter of $0.4 \mathrm{~nm}$ ). On the other hand, this energy shift has never been observed on single PC molecules except if these latter are adsorbed exactly on a defect for example straddling a step. Moreover, the positions of the PC molecules are randomly distributed on the $\mathrm{NaCl}$ surface after evaporation on the cold substrate since diffusion is blocked and molecules adsorb as it is. Then, it appears highly unlikely that these observations are linked to the adsorption site except if one admits that the trimer formation induces three unstable molecule positions that cannot be reached by a single molecule. This result can also be due to different adsorption heights leading to different hybridizations with the surface. In this case, it should be supposed that this mechanism is perfectly progressive over the three objects, leading to a quasi-linear evolution of the molecule-surface distance from molecule 1 to molecule 3 . A more likely interpretation of this phenomenon is to take into account the intermolecular interactions due to the CHO peripheral groups. Such a phenomenon has already been reported for linear oligomers composed of trimers and tetramers connected by hydrogen bonds 32. In this work, the calculations performed in the gas phase show that the electronic characteristics of the connected monomers and particularly the frontier orbitals along the small assemblies are significantly modified by charge transfer. Due to this phenomenon, the HOMO and LUMO states are each located at one end of the chain. For PC molecules, it has been previously demonstrated that charge transfer has been decisive in the trimer formation on the $\operatorname{Ag}(111)$ substrate at ambient temperature (Fig. 2c) and in the adsorption along the steps. Because of the proximity of the three molecules in the row and the decoupling role of the $\mathrm{NaCl}$ layer, it is possible that the electrostatic interactions between polar groups play a significant role and induce a small charge transfer.

To support these assumptions, calculations have also been performed. Three PC molecules in gas phase have been positioned in the same plane at an interatomic distance corresponding to the experimental results (i.e., $1.3 \mathrm{~nm}$ from 


\begin{tabular}{|c|c|c|c|c|}
\hline Energy Level & Single PC & Molecule 1 & Molecule 2 & Molecule 3 \\
\hline HOMO (eV) & -8.33 & -8.50 & -8.26 & -8.07 \\
LUMO (eV) & -1.62 & -1.82 & -1.60 & -1.38 \\
Charge (e) & 0.000000 & 0.002992 & -0.000096 & -0.002895 \\
\hline
\end{tabular}

Table 1: Results of the PM6 calculations in the gas phase for a single PC and for three PC molecules each separated by $1.3 \mathrm{~nm}$ and with the orientation presented in Fig. 8

center to center) and with the same orientation (see Fig. 8). The position of the frontier orbitals has been investigated using a semi-empirical approach and the MOPAC code with the PM6 method [33. The results are reported in Table 1. These calculations clearly show a shift of the energy levels of molecule 1 and 3 as compared to the central molecule or a single molecule. This shift (about $-0.23 \mathrm{eV}$ for 1 and +0.2 for 3 ) is of the same order than the value experimentally obtained $(0.1 \mathrm{eV})$ and with an evolution from molecule 1 to 3 consistent with the STM observations. Note also that the characteristics of the central PC are very close to those of a free molecule.

Thus, the left molecule receives an excess of charge $-\delta q$. As a consequence, the energy difference between the Fermi level and the LUMO state decreases and this state is observed at a lower voltage. On the contrary, the right molecule, which transfers this charge to the central one, looses a charge $-\delta q$. In this case, the $\mathrm{E}_{F}$-LUMO increases and the unoccupied state is observed at a higher voltage. In this process, the central molecule remains uncharged and behaves as expected from the single molecule experiments. This kind of phenomenon is obviously impossible on a metal and is only allowed by the decoupling properties of the thin $\mathrm{NaCl}$ layer.

\section{Conclusion}

In this paper, $\mathrm{PC}$ molecules deposited on a $\mathrm{Ag}(111)$ substrate and on $\mathrm{NaCl}$ ultra-thin layers have been investigated by STM and NC-AFM at low temper- 
320 formation of characteristic trimers at ambient temperature while the observation of single objects is possible when the sample is cooled at $4.5 \mathrm{~K}$. On ultrathin $\mathrm{NaCl}$ layers, it is well known that the molecule shape is related to its molecular orbitals. Using simple STM image calculations, we demonstrate that the localization of the aldehyde group is then possible. The formation of small clusters suggests that these peripheral groups favor the electrostatic interactions and this could slightly change the relative position of the frontier orbitals with respect to the Fermi level. From this point of view, the experiments conducted on the $\mathrm{NaCl}$ layer bring new insigths in the interactions between derivative perylene molecules. At ultra-low coverage, these investigations can help to explain the geometry of the molecular assemblies that take place on a metallic susbtrate or at least illustrate what are the dominant intermolecular forces within these domains.

\section{References}

[1] M. Nakaya, Y. Okawa, C. Joachim, M. Aono, T. Nakayama, ACS Nano 8 (2014) 12259-12264. doi:10.1021/nn504275b.

[2] S. Jan van der Molen, P. Liljeroth, Journal of Physics: Condensed Matter 22 (2010) 133001. doi:10.1088/0953-8984/22/13/133001.

[3] S. Munery, N. Ratel-Ramond, Y. Benjalal, L. Vernisse, O. Guillermet, X. Bouju, R. Coratger, J. Bonvoisin, European Journal of Inorganic Chemistry 2011 (2011) 2698-2705. doi 10.1002/ejic.201100116.

[4] G. Franc, A. Gourdon, Physical Chemistry Chemical Physics 13 (2011) 14283. doi:10.1039/c1cp20700h.

¿ [5] F. Klappenberger, Progress in Surface Science 89 (2014) 1-55. doi 10.1016/ j.progsurf.2013.10.002.

[6] L. Grill, M. Dyer, L. Lafferentz, M. Persson, M. V. Peters, S. Hecht, Nature Nanotechnology 2 (2007) 687-691. doi:10.1038/nnano.2007.346. 
[7] J. A. Lipton-Duffin, O. Ivasenko, D. F. Perepichka, F. Rosei, Small 5 (2009) 592-597. doi $10.1002 / \mathrm{smll} .200801943$.

[8] C. Liu, W. Zhang, Q. Zeng, S. Lei, Chemistry - A European Journal 22 (2016) 6768-6773. doi:10.1002/chem.201601199.

[9] M. Koudia, M. Abel, C. Maurel, A. Bliek, D. Catalin, M. Mossoyan, J.C. Mossoyan, L. Porte, The Journal of Physical Chemistry B 110 (2006) 10058-10062. doi:10.1021/jp0571980.

[10] J.-M. Lehn, Science 295 (2002) 2400-2403. doi 10.1126/science.1071063

[11] J. V. Barth, Annual Review of Physical Chemistry 58 (2007) 375-407. doi $10.1146 /$ annurev . physchem.56.092503.141259.

[12] S. Barlow, R. Raval, Surface Science Reports 50 (2003) 201-341. doi 10. 1016/S0167-5729(03)00015-3.

${ }_{360}^{6}$ [13] J. V. Barth, G. Costantini, K. Kern, Nature 437 (2005) 671-679. doi:10. 1038/nature04166.

[14] B. Calmettes, N. Estrampes, C. Coudret, T. J. Roussel, J. Faraudo,

q R. Coratger, Phys. Chem. Chem. Phys. 18 (2016) 20281-20289. doi:10. 1039/C5CP06733B.

[15] S. Aftergut, H. Cole, Red perylene dichroic dye containing liquid crystal formulations. US Patent 4,378,302 (1983).

[16] S. C. Tse, K. K. Tsung, S. K. So, Applied Physics Letters 90 (2007) 213502. doi:10.1063/1.2740110.

[17] A. Dodabalapur, H. E. Katz, L. Torsi, Advanced Materials 8 (1996) 853855. doi $10.1002 /$ adma.19960081021.

[18] C. W. Tang, Applied Physics Letters 48 (1986) 183-185. doi:10.1063/1. 96937. 
[19] S. Ferrere, B. A. Gregg, New Journal of Chemistry 26 (2002) 1155-1160. doi $10.1039 / \mathrm{b} 203260 \mathrm{k}$.

375 [20] G. Wang, R. Zhang, C. Xu, R. Zhou, J. Dong, H. Bai, X. Zhan,

1. ACS Applied Materials \& Interfaces 6 (2014) 11136-11141. doi 10.1021/ am503171s.

[21] A. Liscio, K. Kouroupis-Agalou, A. Kovtun, E. Gebremedhn, M. El Garah, W. Rekab, E. Orgiu, L. Giorgini, P. Samorì, D. Beljonne, V. Palermo, ChemPlusChem 82 (2017) 358-367. doi 10.1002/cplu.201600503.

[22] R. J. Brea, M. J. Pérez-Alvite, M. Panciera, M. Mosquera, L. Castedo, J. R. Granja, Chemistry - An Asian Journal 6 (2011) 110-121. doi 10. 1002/asia.201000545.

[23] R. Smoluchowski, Physical Review 60 (1941) 661-674. doi 10.1103/ PhysRev.60.661.

[24] L. Gross, F. Mohn, N. Moll, P. Liljeroth, G. Meyer, Science 325 (2009) 1110-1114. doi $10.1126 /$ science. 1176210

[25] L. Gross, F. Mohn, N. Moll, B. Schuler, A. Criado, E. Guitian, D. Pena, A. Gourdon, G. Meyer, Science 337 (2012) 1326-1329. doi 10.1126/ science.1225621.

[26] R. Smoluchowski, Physical Review 60 (1941) 661-674. doi 10.1103/ PhysRev.60.661.

[27] J. Zhang, P. Chen, B. Yuan, W. Ji, Z. Cheng, X. Qiu, Science 342 (2013) 611-614. doi $10.1126 /$ science. 1242603 .

[28] J. Repp, G. Meyer, S. Paavilainen, F. E. Olsson, M. Persson, Physical Review Letters 95 (2005) 225503. doi 10.1103/PhysRevLett.95.225503.

[29] F. Tautz, Progress in Surface Science 82 (2007) 479-520. doi 10.1016/j. progsurf.2007.09.001. 
[30] K. A. Cochrane, A. Schiffrin, T. S. Roussy, M. Capsoni, S. A. Burke, Nature Communications 6 (2015) 8312. doi 10.1038/ncomms9312

[31] H. Aldahhak, W. Schmidt, E. Rauls, Surface Science 641 (2015) 278-281. doi $10.1016 / j$. susc.2015.01.013

[32] H. Tan, W. Qu, G. Chen, R. Liu, The Journal of Physical Chemistry A 109 (2005) 6303-6308. doi 10.1021/jp051444q.

405 [33] D. Young, in: Computational Chemistry: A Practical Guide for Applying Techniques to Real World Problems, Wiley-Interscience, 2001, p. Appendix A. A.3.2. 